\title{
Direct Detection of the L-Dwarf Donor in WZ Sagittae
}

\author{
Thomas E. Harrison ${ }^{1}$ \\ Department of Astronomy, New Mexico State University, Box 30001, MSC 4500, Las Cruces, NM \\ 88003-8001
}

\begin{abstract}
Analysis of a large set of phase-resolved $K$-band spectra of the cataclysmic variable WZ Sge shows that the secondary star of this system appears to be an L-dwarf. Previous $K$-band spectra of WZ Sge found that the CO overtone bandheads were in emission. We show that absorption from the ${ }^{12} \mathrm{CO}_{(2,0)}$ bandhead of the donor star creates a dip in the ${ }^{12} \mathrm{CO}_{(2,0)}$ emission feature. Measuring the motion of this feature over the orbital period, we construct a radial velocity curve that gives a velocity amplitude of $\mathrm{K}_{\mathrm{abs}}=$ $520 \pm 35 \mathrm{~km} \mathrm{~s}^{-1}$, consistent with the previously published values for this parameter.
\end{abstract}

Key words: infrared: stars — stars: cataclysmic variables — stars: individual (WZ Sagittae)

${ }^{1}$ Visiting Observer, W. M. Keck Observatory, which is operated as a scientific partnership among the California Institute of Technology, the University of California, and the National Aeronautics and Space Administration. NOAO Prop. \#2004B-0093

\section{Introduction}

Cataclysmic variables (CVs) are short period binaries where the white dwarf primary is accreting matter from a lower mass secondary star. An angular momentum loss process keeps the system in contact (c.f., King \& Kolb 1995), and the orbital period shortens over time as the secondary continues to lose mass. The standard evolutionary paradigm for CVs proposes that as these systems evolve to shorter and shorter periods, they eventually reach a period minimum somewhere between 70 and 80 minutes (Kolb \& Baraffe 1999, Howell et al. 1997, Knigge et al. 2011, and references therein). At this orbital period, the donor star has too low of a mass to support core hydrogen burning. After this point, the secondary can no longer adjust to the mass loss on its thermal time scale, and will expand upon further mass loss. The orbital period now must increase. This sudden change to longer orbital periods has been termed the "period bounce." One of the early lists for period bouncer candidates was compiled by Patterson (1998). Patterson investigated the mass ratio distribution versus orbital period for the shortest period CVs, and found four good candidates for being period bouncers; one of these is WZ Sge.

WZ Sge is short period CV that exhibits infrequent, but large amplitude dwarf novae outbursts (Patterson et al. 2002). Whereas other short period CVs with similar orbital periods have outbursts 
on the weeks to months timescale, WZ Sge only has outbursts on a timescale of decades. The orbital period of WZ Sge is accurately known from bright spot eclipse events $\left(\mathrm{P}_{\text {orb }}=0.056687846\right.$ d; Patterson et al. 1998). The presence of "superhumps" in its outburst light curve (Patterson et al. 2002) is taken as evidence for a small mass ratio, if the thermal-tidal instability theory of Osaki (1996) is correct. Assuming an appropriate mass for the white dwarf suggests a secondary star close to the stellar/sub-stellar boundary. Such an object should have prominent CO absorption features in its $K$-band spectra, but to date, such observations have revealed that WZ Sge instead exhibits $C O$ emission features whose origin appears to be the accretion disk (Howell et al. 2004). Thus, even though WZ Sge is the closest known CV at 43 pc (Thorstensen 2003, Harrison et al. 2004a) and has a very low accretion rate (Patterson 1998), the donor object has remained elusive.

While direct photospheric absorption features from the donor in WZ Sge have not previously been detected, $\mathrm{H} \alpha$ emission, due to the irradiation of the companion during the 2001 outburst, were clearly seen, and led to the first quantitative constraints on the nature of the secondary. Steeghs et al. (2001) found that the narrow $\mathrm{H} \alpha$ emission feature leads to a radial velocity semi-amplitude of $\mathrm{K}_{\mathrm{em}}=493 \pm 10 \mathrm{~km} \mathrm{~s}^{-1}$ for the donor. Modeling the same data set gives $\mathrm{K}_{1}=37 \pm 5 \mathrm{~km} \mathrm{~s}^{-1}$. Incorporation of systematic errors leads to $493 \mathrm{~km} \mathrm{~s}^{-1} \leq \mathrm{K}_{\mathrm{em}} \leq 585 \mathrm{~km} \mathrm{~s}^{-1}$, and a mass ratio range of $0.04 \leq q \leq 0.075$. With $\mathrm{M}_{1}>0.70 \mathrm{M}_{\odot}$, the secondary has $\mathrm{M}_{2}<0.11 \mathrm{M}_{\odot}$, consistent with an object near the stellar/substellar boundary (Dupuy et al. 2010). Harrison et al. (2013) modeled infrared light curves and optical/IR photometry of WZ Sge and found that both suggest a secondary with a temperature near $\mathrm{T}_{\text {eff }}=1800 \mathrm{~K}$. They found that an L2 dwarf (with $\mathrm{M}_{\mathrm{K}}=$ 11.1) that supplied $\sim 60 \%$ of the $K$-band flux was consistent with the infrared light curves.

Given these results, the secondary star of WZ Sge should be visible in $K$-band spectra if it is of normal composition. However, with $\mathrm{CO}$ emission, and the possibility of sub-solar carbon abundances (see Harrison \& Hamilton 2015), a more extensive set of data is required to search for the donor. We describe below a large set of $K$-band spectra that clearly reveal the L-dwarf secondary in WZ Sge. In the next section we describe the observations, in section 3 we detail our analysis and results, and draw our conclusions in section 4 .

\section{Observations}

$K$-band spectroscopy of WZ Sge was obtained using NIRSPEC on Keck II on the night of 2004 August 28. We used NIRSPEC in single order, low resolution mode with the 0.38 " slit, resulting in a dispersion of $4.23 \AA /$ pix $(\mathrm{R}=2300)$. WZ Sge was observed continuously from 05:23 UT to 07:37 UT and from 08:20 UT until 11:30 UT. The 43 minute gap is due to the fact that WZ Sge transits the zenith as seen from Mauna Kea, and thus cannot be tracked by an alt-az telescope. We were on source for $5.4 \mathrm{hr}$, obtaining near complete coverage of four orbital periods. Eighty eight spectra were obtained. The exposure times for all of the WZ Sge spectra were $180 \mathrm{~s}$. The raw spectra have $\mathrm{S} / \mathrm{N} \sim 30$ in the brightest portions of the continuum. Before observing WZ Sge, during the transit, and immediately afterwards, arc lamps, flats and telluric standard star data were obtained. 
The observations were reduced in the normal way using IRAF. Bright G2V stars were observed to provide for telluric correction for the wide range of airmasses covered by our observations of WZ Sge $(1.53 \leq \sec z \leq$ to 1.0$)$. We used G2V telluric standards in an attempt to better recover the $\mathrm{H} \mathrm{I} \mathrm{Br} \gamma$ profile, using the procedure outlined in Maiolino et al. (1996): After division by the G2V telluric, the spectrum is then multiplied by a rotationally convolved solar spectrum (see Harrison

et al. 2004b). The data were phased to the ephemeris of Patterson et al. (1998), but with the offset in the timing of inferior conjunction found by Harrison et al. (2013).

\section{Results}

In Fig. 1 we present a subset of the data at the phases $0.0,0.25,0.5$ and 0.75 for comparison with the $K$-band spectra presented in Howell et al. (2004). The CO emission features appear to be of the same strength for both epochs, however, emission from $\mathrm{H}_{2}$ is not present in the new data. The main difference between the two data sets is the dramatic improvement in $\mathrm{S} / \mathrm{N}$ afforded by having numerous redundant spectra to median together. The phase 0.0 spectrum clearly shows an absorption dip centered in the middle of the first $\mathrm{CO}_{(2,0)}(\lambda 2.294 \mu \mathrm{m})$ emission feature. At phase 0.25 , the red side of the $\mathrm{CO}$ emission feature appears to dramatically decline as would be expected if the underlying absorption feature of the secondary star was maximally red shifted at this time. The reverse happens at phase 0.75: the blue wing of the CO emission feature is now greatly weakened. The changing morphology of the $\mathrm{CO}$ emission feature in Fig. 1 suggests the presence of a late-type secondary.

\subsection{A Radial Velocity Curve for the Donor}

To more closely examine the CO region, we present Fig. 2, The spectra shown in this figure are the medians of 7 to 11 individual spectra with $\Delta \phi= \pm 0.05$ in phase. This figure has data at every 0.1 in phase, with the addition of the $\phi=0.25$ (red) and 0.75 (blue) spectra shown in Fig. 1 . The bandhead location for $\mathrm{CO}_{(2,0)}$ is delineated by the dotted line. As is clear, the absorption dip is centered in the emission feature at phase 0 , moves redward to a maximum at phase 0.25 , returns to no offset at phase 0.5 , and exhibits the maximum blueshift at phase 0.75 .

To obtain a radial velocity curve, we rebin the data in $\Delta \phi= \pm 0.025$ increments to reduce orbital smearing. We also average the phases of the individual spectra to get a weighted mean value for the phase of each medianed data set. We used the line measurement capability in IRAF ("splot") to fit a Gaussian profile to the observed absorption feature. The radial velocity curve we derive, shown in Fig. 3, consists of 20 individual measurements. To arrive at this result, we subtracted the measured wavelength of the $\mathrm{CO}$ bandhead in the individual spectra from its expected position $(2.294 \mu \mathrm{m})$. We then offset the data points by the mean of the entire radial velocity data set. In these lower $\mathrm{S} / \mathrm{N}$ spectra, the location of the first overtone $\mathrm{CO}$ bandhead is often poorly 
defined, leading to significant deviations from a smooth radial velocity curve. The origin of the most deviant measurements can be clearly traced back to the data shown in Fig. 2 (such as near $\phi=0.6)$. With the expectation of a sinewave, we performed a $\chi^{2}$ analysis of the data presented in Fig. 3. The result, shown in Fig. 44 finds a best fit velocity of $\mathrm{K}_{\mathrm{abs}}=520 \pm 35 \mathrm{~km} \mathrm{~s}^{-1}$. This value for $K_{a b s}$ is consistent with the range of velocities derived by Steeghs et al. (2001). A radial velocity curve with this amplitude is plotted in red in Fig. 3. The $\chi^{2}$ fitting also allows us to assign error bars to our measurements, as to get values of $\chi_{\text {red }}^{2} \sim 1$, requires $\sigma=108 \mathrm{~km} \mathrm{~s}^{-1}$. This value for the error on the individual radial velocity measurements appears to represent the observed scatter quite well.

The exact value of the semi-amplitude of the secondary is difficult to constrain. Our exposures were three minutes in duration $(\Delta \phi=0.04)$, and our medianed spectra spanned $\Delta \phi \leq 0.05$. Thus, the short period of WZ Sge introduces orbital smearing into our results. Combined with our large error bars, the derived value of $\mathrm{K}_{\mathrm{abs}}$ is not especially precise. Careful examination of Fig. 3 reveals that the absorption features in the continuum between $\mathrm{H} \mathrm{I} \mathrm{Br} \gamma$, and the first overtone bandhead of $\mathrm{CO}$, also track the motion of the secondary. These features closely resemble those of an L2 dwarf. If we assume that these are from the donor star, we can endeavour to determine an alternative value for $\mathrm{K}_{\mathrm{abs}}$. We attempted to use a cross-correlation technique using the L2 dwarf Kelu 1 (obtained from the IRTF Spectral Library'1. Cushing 2005), as our template. Unfortunately, that effort was unsuccessful. We next decided to Doppler-correct the entire data set until these continuum features were at their sharpest. We performed a $\chi^{2}$ test over the velocity interval $490 \leq \mathrm{K}_{2} \leq 575 \mathrm{~km} \mathrm{~s}^{-1}$, and over the wavelength range $2.19 \leq \lambda \leq 2.28 \mu \mathrm{m}$. The results, however, were ambiguous, with nearly identical values of $\chi^{2}$ throughout the range. The strongest minimum was at $\mathrm{K}_{\mathrm{abs}}=510 \mathrm{~km}$ $\mathrm{s}^{-1}$, with a secondary minimum at $\mathrm{K}_{\mathrm{abs}}=550 \mathrm{~km} \mathrm{~s}^{-1}$. A visual inspection suggests that $\mathrm{K}_{2}=555$ $\mathrm{km} \mathrm{s}^{-1}$ produces a spectrum whose features best match those of Kelu 1.

In Fig. 5, we compare the Doppler-corrected data set (assuming $\mathrm{K}_{2}=555 \mathrm{~km} \mathrm{~s}^{-1}$ ) to Kelu 1. Kelu 1 is in fact a binary consisting of $\sim \mathrm{L} 2$ and $\sim \mathrm{L} 3$ dwarfs (Liu \& Leggett 2005). The primary is about 0.5 mag brighter in the $K$-band than the secondary. Kelu 1 is a rapid rotator, with a period of $\mathrm{P}_{\text {rot }}=1.8 \mathrm{hr}$ (Clarke et al. 2002), and $v \sin i=60 \pm 5 \mathrm{~km} \mathrm{~s}^{-1}$ (Basri et al. 2000). With $\mathrm{K}_{2}=$ $555 \mathrm{~km} \mathrm{~s}^{-1}$, and the mean parameters for WZ Sge from Steeghs et al. (2001), the rotation velocity of the secondary should be of order $v \sin i \sim 85 \mathrm{~km} \mathrm{~s}^{-1}$. Neither rotational velocity is resolved in these spectra, explaining their similarity in appearance. To construct Fig. 5 we continuum fit and divided the spectra of both objects. In addition, we then divided the spectrum of Kelu 1 by a factor of 2.0 to best match the amplitude of the corresponding features in WZ Sge. This amount of "dilution" is consistent with that found in the light curve modeling of WZ Sge by Harrison et al. (2013).

\footnotetext{
${ }^{1}$ http://irtfweb.ifa.hawaii.edu/ spex/IRTF_Spectral_Library/
} 


\subsection{The CO Features of the L Dwarf Donor}

In the mean of the full data set, the first overtone CO feature in WZ Sge certainly appears to be much weaker than that seen in Kelu 1. As noted in Harrison et al. (2013), light curve modeling indicates irradiation of the donor star near $\phi=0.5$. In Fig. 5 we also plot a portion of the Doppler-corrected data set centered on $\phi=0.0$. The CO features are considerably stronger at inferior conjunction perhaps partly due to the lack of irradiation at this phase, but mostly due to the dramatic decrease in the dilution from the partial eclipse of the accretion disk and hotspot. The light curve modeling in Harrison et al. (2013) can provide insight on the level of irradiation. In their ellipsoidal models, the secondary minimum $(\phi=0.5)$ in the model $K$-band light curve (their Fig. 12) is $0.2 \mathrm{mag}$ less deep than it would be if there was no irradiation. If there is no irradiation, and the secondary dominated the $K$-band luminosity, the secondary minimum would be slightly deeper than the primary minimum (c.f., Fig. 14 in Harrison \& Campbell 2015). Thus, if the secondary minimum is weaker than the primary minimum, it is an indication of irradiation. The change in temperature required to create the observed $0.2 \mathrm{mag}$ difference, however, is very small $(\sim 5 \%)$. This is insufficient to significantly change the spectral type of the donor and thus alter the strength of the $\mathrm{CO}$ feature.

We believe that there are two plausible scenarios that might explain why the $\mathrm{CO}$ absorption feature in the donor is especially weak near $\phi=0.5$. The first is that the CO emission appears to be strongest at this phase. If one compares the entire set of $\mathrm{CO}$ emission features they are all strongest at this phase. Why does this happen? It could be that irradiation from the hotspot is suppressing $\mathrm{CO}$ emission from nearby regions in the accretion disk. Since the hotspot leads the secondary by $\Delta \phi \sim 0.05$, if there is a decrease of $\mathrm{CO}$ emission near the hotspot, the red wing of the $\mathrm{CO}$ emission features on the receding side of the disk near the hotspot would be diminished, and the blue wing would be lessened on the approaching part of its orbit. Near phase 0.5, there would be no significant affect, and the profiles would be at their strongest, and most symmetric. The morphology of the $\mathrm{CO}$ emission features shown in Fig. 2 are certainly consistent with this scenario. Note also that the center of the first overtone emission feature suggests that it is slightly redshifted at phase 0.5 , and that the red wing of the same feature is weaker at phase 0 . The small offset in phase of the hotspot certainly could explain the changing morphologies of the emission features. This phasing offset would also produce an asymmetric irradiation of the secondary star. There might be evidence for this in the infrared light curves of WZ Sge. There is a weak excess in the $K$-band and Spitzer IRAC $4.5 \mu \mathrm{m}$ bandpass light curves of WZ Sge at $\phi=0.7$ (vs. 0.2), suggesting a tiny bit of additional irradiation of the hemisphere facing the observer at this time. This amount of irradiation appears to be much too small, however, to impart a significant change on the spectral properties of the secondary.

Secondly, it might be possible for the hotspot to obscure the secondary near superior con-

junction. As noted by a number of authors (see Mason et al. 2000 and references therein), the hotspot in WZ Sge has considerable optical depth. If we assume this remains true in the $K$-band, and that the hotspot has sufficient vertical extent, it might be able to obscure a small portion of 
the secondary star near $\phi=0.5$. The $K$-band light curve in Harrison et al. (2013) does show a sharper than expected secondary minimum. The presence of strong CO emission and a small amount of obscuration could combine to create the weaker than expected $\mathrm{CO}$ absorption features near superior conjunction.

We can attempt to model the depth of the $\mathrm{CO}$ absorption feature seen in the medianed full data set by adding a CO emission spectrum to the L2 data following the procedure used in Harrison \& Hamilton (2015, see their Fig. 21). In the case of WZ Sge, there is the expectation that the CO features will have a double-peaked profile similar to the H I and He I lines. Since it is impossible to know the exact splitting a priori, we model several cases. The two peaks of the H I lines (see Fig. 1) are separated by $105 \AA$. If the CO emission comes from an outer, cooler region of the disk, the splitting should be less than this. A single Gaussian fit to the $\mathrm{CO}_{(2,0)}$ feature leads to an estimate of the velocity broadening of $\sim 1300 \mathrm{~km} \mathrm{~s}^{-1}$. In Fig. 6, we have added a $\mathrm{CO}$ emission line spectrum with this amount of velocity broadening to the IRTF spectrum of Kelu 1. Next we add two velocity-broadened CO emission spectra shifted by $\pm 25 \AA$ (total $\Delta \lambda=50 \AA$ ) to the L2 dwarf spectrum, and plot it just below the single $\mathrm{CO}$ emission line spectrum. Below that is a $\mathrm{CO}$ emission spectrum also shifted by $\pm 25 \AA$, but with each emission component broadened by 650 $\mathrm{km} \mathrm{s}^{-1}$. As shown in Harrison \& Hamilton (2015), as the Gaussian profile of the CO emission narrows, the observed depth of the CO feature decreases due to the emission feature contributing more flux into the deepest parts of the absorption bandhead of the secondary star. Note that at this velocity, the blue wing does not match the observations. Below this spectrum in Fig. 6 we plot the sum of a $\mathrm{CO}$ emission spectrum and the L2 dwarf where the separation between the two peaks is $\Delta \lambda= \pm 50 \AA$, and below that, one with $\pm 75 \AA$ separation. At a velocity broadening of 1300 $\mathrm{km} \mathrm{s}^{-1}$, splittings of $\leq \pm 50 \AA$ are too small to actually affect the CO emission profile. As we move to larger shifts between the CO emission peaks, the bandhead minima absorb the peak of the redshifted emission components. The sum result of this modeling process is that we can reproduce the observed $\mathrm{CO}$ absorption feature, and that the two peaks of the $\mathrm{CO}$ feature must be shifted by less than $\Delta \lambda= \pm 50 \AA$. Unfortunately, the large and uncertain velocity broadening does not allow us to put useful constraints on the location of the $\mathrm{CO}$ emission region.

As shown in Harrison \& Hamilton (2015), carbon is highly deficient in the long period CVs SS Cyg, RU Peg, and GK Per (and by inference, the numerous other CVs with weak CO features seen in the $K$-band spectroscopic surveys of Harrison et al. 2004b, 2005). In contrast, Hamilton et al. (2011) found that the secondary stars in several CVs with short orbital periods similar to that of WZ Sge appeared to have normal CO absorption strengths. They also found that when corresponding UV data exists, CVs with weak CO features in the $K$-band had white dwarfs with apparent deficits of carbon. Analysis of the minimum light UV spectrum of WZ Sge by Cheng et al. (1997) found that the white dwarf appears to have a super-solar abundance of carbon. Our results for WZ Sge indicate a donor with normal levels of carbon.

Given that we have matched the orbitally averaged spectrum of WZ Sge to an L2 dwarf, and there is strong evidence for irradiation, the actual spectral type of the donor must be later than L2. 
To attempt to better constrain the spectral type of the secondary star we more closely examine the $K$-band spectra of L dwarfs. In Fig. 7, we plot spectra from the IRTF Spectral Library for spectral types from M8 to L8. The lack of a Na I doublet $(2.2 \mu \mathrm{m})$ at any phase during the orbit confirms that the donor must have a spectral type later than L1. The strengths of the CO features do not change significantly until the latest L dwarfs. The reason for this is the small temperature change from one subtype to the next. As shown in Basri et al. (2000), the temperature decrease from L0 to L4 is only $\Delta \mathrm{T}=350 \mathrm{~K}$. By L5, the ${ }^{12} \mathrm{CO}_{(3,1)}$ bandhead (at $2.322 \mu \mathrm{m}$ ) has become much weaker, and is very weak at L8. It is clear that in the $\phi=0.0$ spectrum (Fig. 5), the ${ }^{12} \mathrm{CO}_{(3,1)}$ bandhead is quite strong. The remainder of the main absorption features in the continua of $\mathrm{L}$ dwarfs (or opacity minima) show much smaller changes with decreasing temperature. We conclude that the spectral type of the secondary in WZ Sge is later than L2, but is earlier than L8. Using the temperature scale from Basri et al. (2000) suggests a temperature for the donor of between 1800 and $2000 \mathrm{~K}$, consistent with the results of Harrison et al. (2013).

\subsection{The Masses of the Components in WZ Sge}

With the expectation that an ultra-short period CV like WZ Sge is an older object, and that its companion was once a more massive star, it is unclear how closely the donor object properties will match those of a field brown dwarf. Since such objects have no internal nuclear energy source, the radii of isolated brown dwarfs depend on their age. As discussed in Sorahana et al. (2013), older brown dwarfs with temperatures near $2000 \mathrm{~K}$ have radii near $0.9 \mathrm{R}_{\text {Jupiter }}$, and are expected to have masses of $0.07 \mathrm{M}_{\odot}$. If we assume this is true for WZ Sge, we can attempt to further constrain the parameters of the system. If $\mathrm{K}_{1} \leq 37 \mathrm{~km} \mathrm{~s}^{-1}$ (Steeghs et al. 2001) and $\mathrm{K}_{\mathrm{abs}} \geq 520 \mathrm{~km} \mathrm{~s}^{-1}$, then $q \leq 0.071$, and $\mathrm{M}_{1} \geq 0.98 \mathrm{M}_{\odot}$. A more precise value for $\mathrm{K}_{1}$, or a measurement of $v \sin i$ for the donor, is desperately needed to further constrain this system.

\section{Discussion and Conclusions}

We have obtained a large set of $K$-band spectra of WZ Sge. When these data are phased to the orbital period, it becomes obvious that the central dip in the CO emission features moved with orbital phase in a pattern that suggested absorption by an underlying source. Measuring the position of the ${ }^{12} \mathrm{CO}_{(2,0)}$ bandhead leads to a radial velocity curve that has a semi-amplitude of $\mathrm{K}_{\mathrm{abs}}=520 \pm 35 \mathrm{~km} \mathrm{~s}^{-1}$. This result is consistent with the range of $\mathrm{K}_{\mathrm{em}}$ values derived by Steeghs et al. (2001) for the motion of the secondary star from observations of the irradiation-induced $\mathrm{H} \alpha$ emission line. When the entire data set is phased using our value of $\mathrm{K}_{2}$, the continuum in the spectrum has features that indicate the donor has a spectral type near L2. The late spectral

type of the donor in WZ Sge, coupled with its short orbital period, suggest that WZ Sge might be a period bouncer (Patterson 1998). Such a classification, however, remains difficult to prove. For example, Knigge et al. (2011) suggest that such objects would have very low masses: $\mathrm{M}_{2} \leq$ 
$0.05 \mathrm{M}_{\odot}$. If spectral type correlates with mass for these objects, the donors in period-bounce CVs would be expected to have the spectral characteristics of late-L/early-T brown dwarfs. The L2 L5 spectral type we have found for WZ Sge would be too early, and cast doubt on its identification as a period bounce system.

While there have been a large number of claims for brown dwarf donors in CVs, there is only one secure case for the direct spectroscopic detection of the photospheric features of a brown dwarf secondary that we are aware of, and that is for the secondary in SDSS J1433+1011 (Littlefair et al. 2013). The donor in SDSS J1433+1011 also has a spectral type near L2, and is a system that has an orbital period very close to the theoretical minimum ( $\left.\mathrm{P}_{\text {orb }}=78.1 \mathrm{~min}\right)$. The best previous example was the polar J121209.31+013627.7, where low resolution infrared spectroscopy is certainly consistent with an L8 donor (Farihi et al. 2008). In the other systems with suspected brown dwarf donors, it is infrared photometry, eclipses and/or radial velocity studies which suggest a sub-stellar companion. Such studies are often dependent on the mass and/or temperature of the white dwarf primary, which remain challenging to constrain. In the case of WZ Sge, we now have two independent radial velocity studies that are consistent with each other and that suggest a sub-stellar donor mass, a light curve modeling program that predicted an L2 donor, and the subsequent direct detection of this object.

An examination of the near-IR spectra of $\mathrm{L}$ dwarfs indicates that the $J$-band is a much better wavelength regime for classification than in the $K$-band. $J$-band spectroscopy was used by Littlefair et al. (2013) to detect the L2 donor in SDSS J1433+1011. As shown in McLean et al. (2000), very strong absorption features of $\mathrm{K} \mathrm{I}$ and $\mathrm{FeH}$ are present that would allow for a more accurate spectral typing of the secondary, and a radial velocity curve could be more easily generated from these lines as they would be unhampered by H I or molecular emission. WZ Sge has in fact been observed in the $J$-band by Littlefair et al. (2000), but the data they presented was not Doppler-corrected, and thus any absorption features from the donor would be washed-out due to its large motions. Phasing that data set would be useful to confirm our results.

TEH is partially supported by a grant from the NSF (AST-1209451). Data presented herein were obtained at the W. M. Keck Observatory, which is operated as a scientific partnership among the California Institute of Technology, the University of California, and NASA. The Observatory was made possible by the generous financial support of the W. M. Keck Foundation. 


\section{References}

Basri, G., Mohanty, S., Allard, F., Hauschildt, P. H., et al. 2000, ApJ, 538, 363

Cheng, F. H., Sion, E. M., Szkody, P., \& Huang, M. 1997, ApJ, 484, L149

363

Clarke, F. J., Tinney, C. G., \& Covey, K. R. 2002, MNRAS, 332, 361

Cushing, M. C., Rayner, J. T., Vacca, W. D. 2005, ApJ, 623, 1115

Dupuy, T. J., Liu, M. C., Bowler, B. P., Cushing, M. C., et al. 2010, ApJ, 721, 1725

Farihi, J., Burleigh, M. R., \& Hoard, D. W. 2008, ApJ, 674, 421

Hamilton, R. T., Harrison, T. E., Tappert, C., Howell, S. B. 2011, ApJ, 728, 16

Harrison, T. E., \& Hamilton, R. T. 2015, AJ, in press (arXiv:1509.03664)

Harrison, T. E., Hamilton, R. T., Tappert, C., Hoffman, D. I., et al. 2013, AJ, 145, 19

Harrison, T. E., Osborne, H. L., \& Howell, S. B. 2005, AJ, 129, 2400

Harrison, T. E., Johnson, J. J., McArthur, B. E., Benedict, G. F., et al. 2004a, AJ, 127, 460

Harrison, T. E., Osborne, H. L., \& Howell, S. B. 2004b, AJ, 127, 3493

Howell, S. B., Harrison, T. E., \& Szkody, P. 2004, ApJ, 602, L49

Howell, S. B., Rappaport, S., \& Politano, M. 1997, MNRAS, 287, 929

King, A. R., \& Kolb, U. 1995, ApJ, 439, 330

Knigge, C., Baraffe, I., \& Patterson, J. 2011, ApJS, 194, 28

Kolb, U., \& Baraffe, I. 1999, MNRAS, 309, 1034

Littlefair, S. P., Savoury, C. D. J., Dhillon, V. S., Marsh, T. R., et al. 2013, MNRAS, 431, 2820

Littlefair, S. P., Dhillon, V. S., Howell, S. B., \& Ciardi, D. R. 2000, MNRAS, 313, 117

Liu, M. C., \& Leggett, S. K. 2005, ApJ, 634, 616

Maiolino, R., Rieke, G. H., \& Rieke, M. J. 1996, AJ, 111, 537

McLean, I. S., Mavourneen, K. W., Becklin, E. E., Figer, D. F., et al. 2000, ApJ, 544, L45

Osaki, Y. 1996, PASP, 108, 39

Paczyński B., 1981, Acta Astr., 31, 1

Patterson, J., Gianluca, M., Richmond, M. W., Martin, B., et al. 2002, PASP, 114, 721

Patterson, J., Richman, H., \& Kemp, J. 1998, PASP, 110, 403

Patterson, J. 1998, PASP, 110, 1132

Skidmore, W., Wynn, G. A., Leach, R., \& Jameson, R. F. 2002, MNRAS, 336, 1223

Sorahana, S., Yamamura, I., \& Murakami, H. 2013, ApJ, 767, 77

Spruit, H. \& Rutten, R. 1998, MNRAS, 299, 768

Steeghs, D., Marsh, T., Knigge, C., Maxted, P. F. L., et al. 2001, ApJ, 562, L145

Thorstensen, J. R., 2003, AJ, 126, 3017 


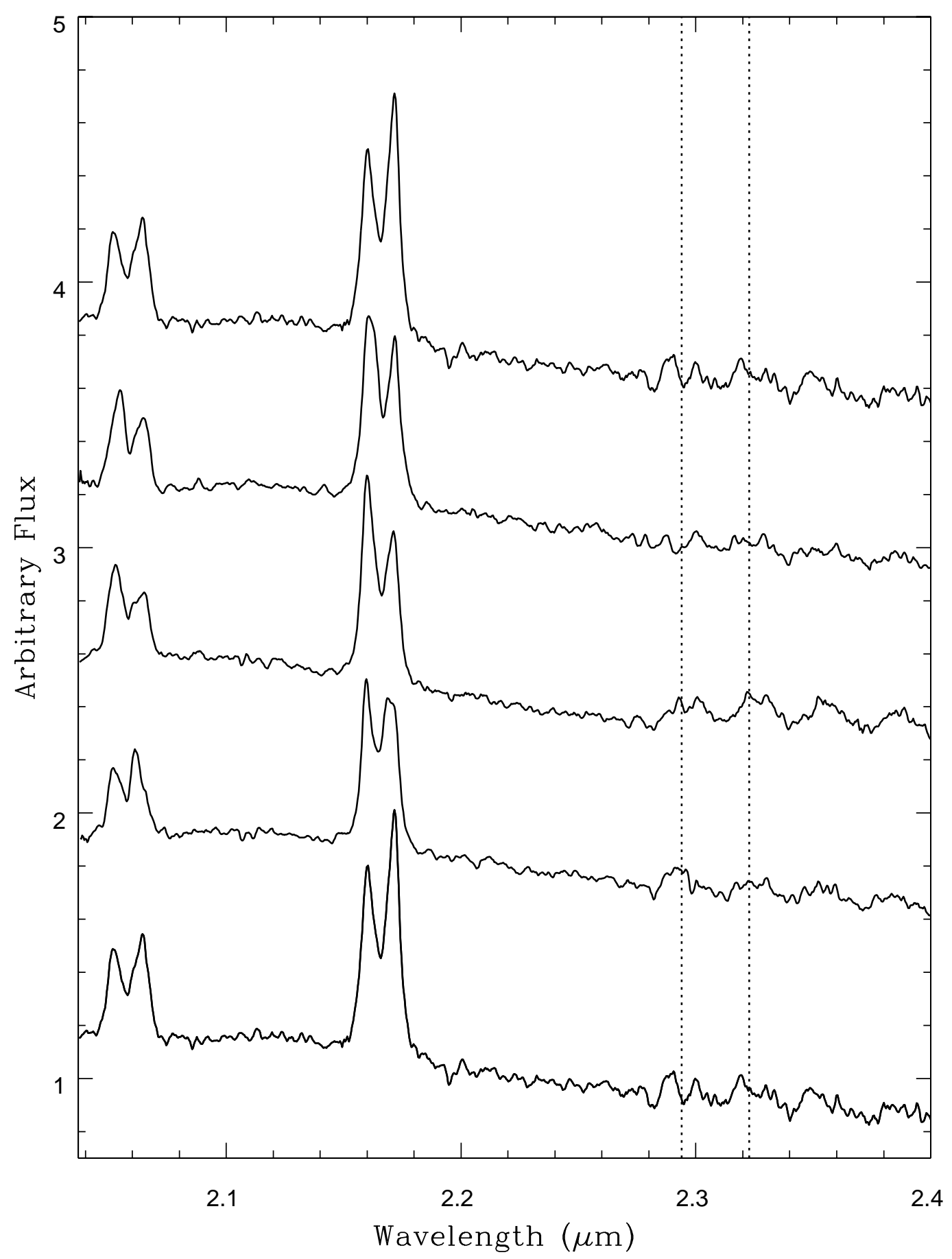

Fig. 1. - The $K$-band spectra of WZ Sge at $\phi=0.0$ (bottom), 0.25, 0.5, 0.75 and 0.0 (top). The spectra presented in this figure have been boxcar smoothed by 5 pixels. The location of the ${ }^{12} \mathrm{CO}_{(2,0)}(\lambda 2.294 \mu \mathrm{m})$ and ${ }^{12} \mathrm{CO}_{(3,1)}(\lambda 2.323 \mu \mathrm{m})$ bandheads are delineated by vertical dotted lines. 


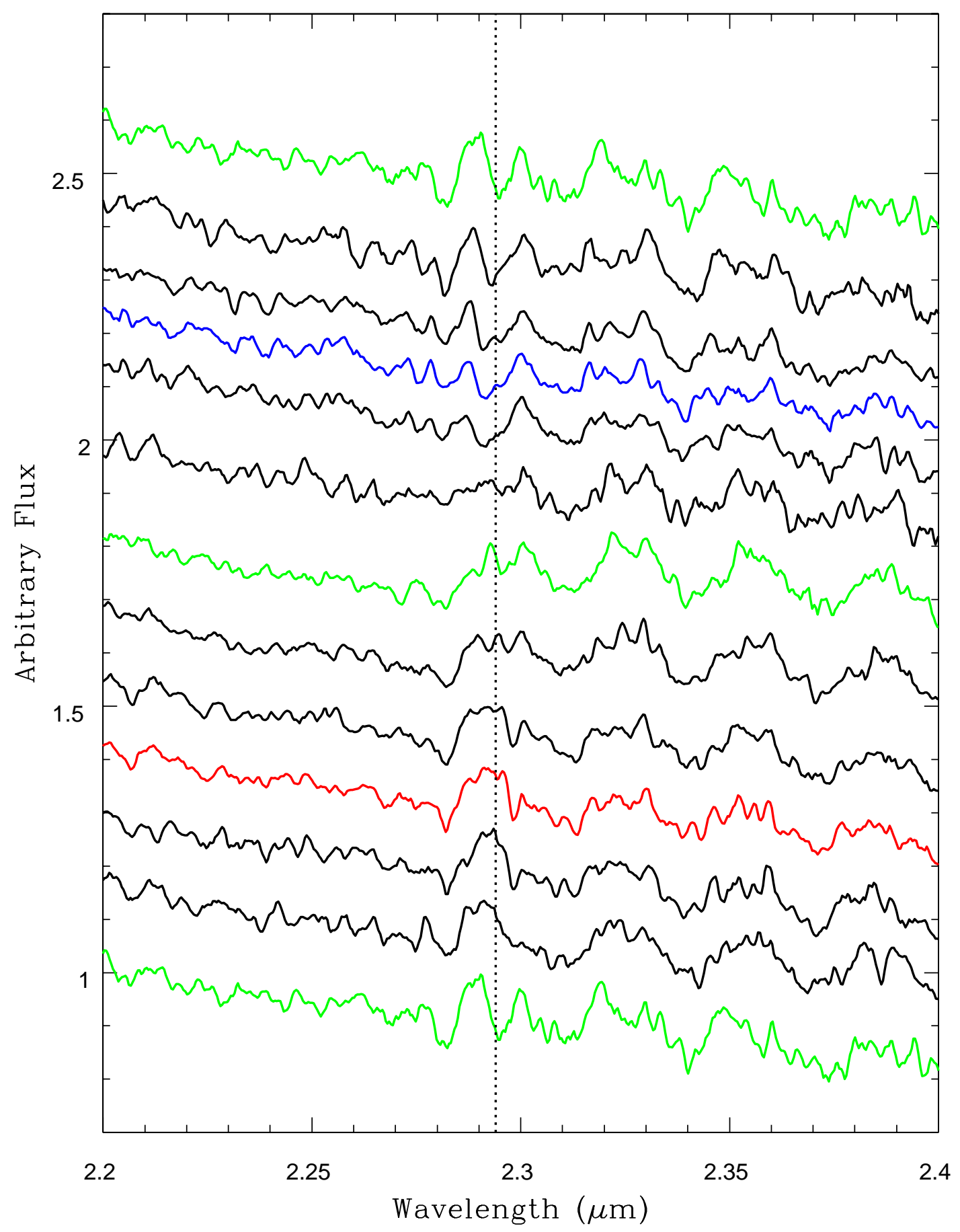

Fig. 2. - The spectra of WZ Sge in the wavelength interval $2.2 \leq \lambda \leq 2.4 \mu \mathrm{m}$. Phase 0 is at the bottom (green), and the spectra step by $\Delta \phi=0.1$, except with the $\phi=0.25$ (red) and 0.75 (blue) spectra inserted into the sequence. The $\phi=0.5$ spectrum is also plotted in green. The spectra presented in this figure have been boxcar smoothed by 5 pixels. The vertical dotted line delineates the location of the ${ }^{12} \mathrm{CO}_{(2,0)}$ bandhead at $2.294 \mu \mathrm{m}$. 


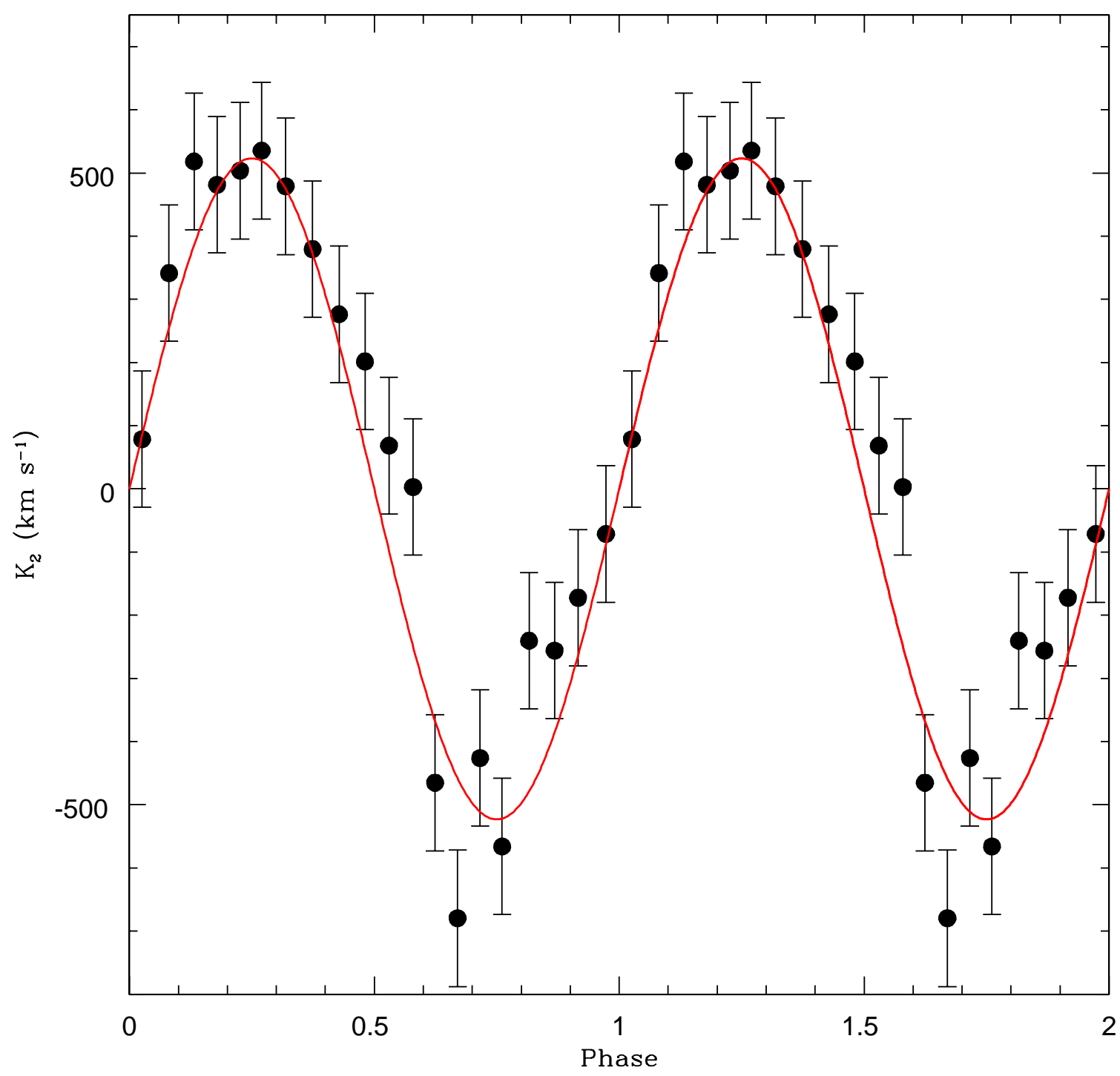

Fig. 3. - The radial velocity measurements of the donor star derived from the positions of the ${ }^{12} \mathrm{CO}_{(2,0)}$ bandhead. A sinusoid with an amplitude of $520 \mathrm{~km} \mathrm{~s}^{-1}$ is plotted in red. 


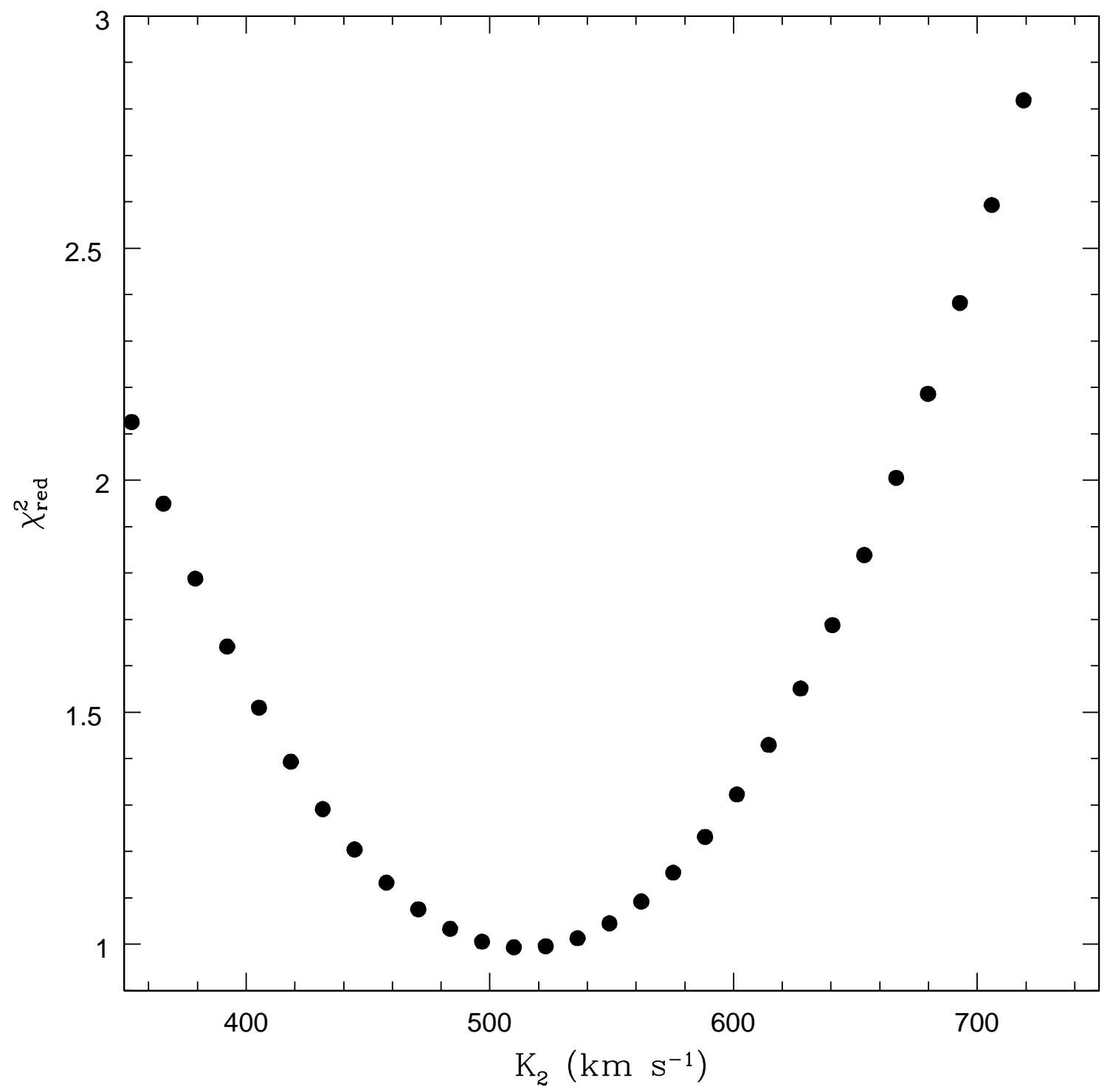

Fig. 4.- A $\chi^{2}$ analysis of the radial velocity data finds that the best fitting $\mathrm{K}_{2}$ radial velocity semi-amplitude is near $520 \mathrm{~km} \mathrm{~s}^{-1}$. 


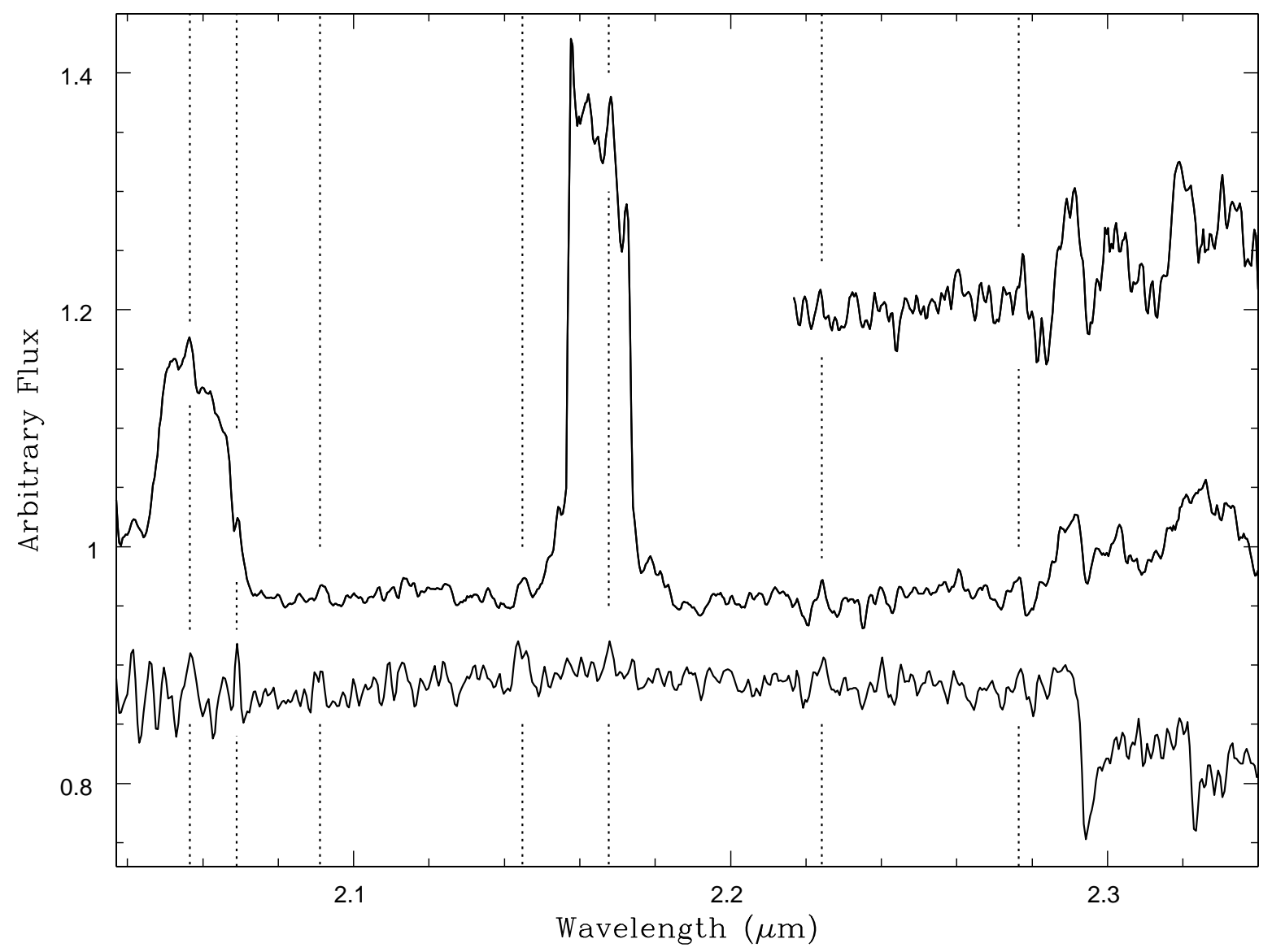

Fig. 5. - The median of the entire data set for WZ Sge Doppler-corrected to $\mathrm{K}_{2}=555 \mathrm{~km} \mathrm{~s}^{-1}$, compared to the spectrum of the L2 dwarf Kelu 1. The vertical dotted lines locate opacity minima peaks in the L2 dwarf that are obviously present in the spectrum of WZ Sge. Note that both have been continuum subtracted, and the L2 dwarf spectrum then divided by a factor of two. On the top-right we plot a segment of the median of the spectra at phase $0.0(\Delta \phi= \pm 0.05)$. The CO features are much stronger near phase 0.0 due to the lack of irradiation, and the lower amount of dilution due to the partial eclipse of the white dwarf and accretion disk at this time. 


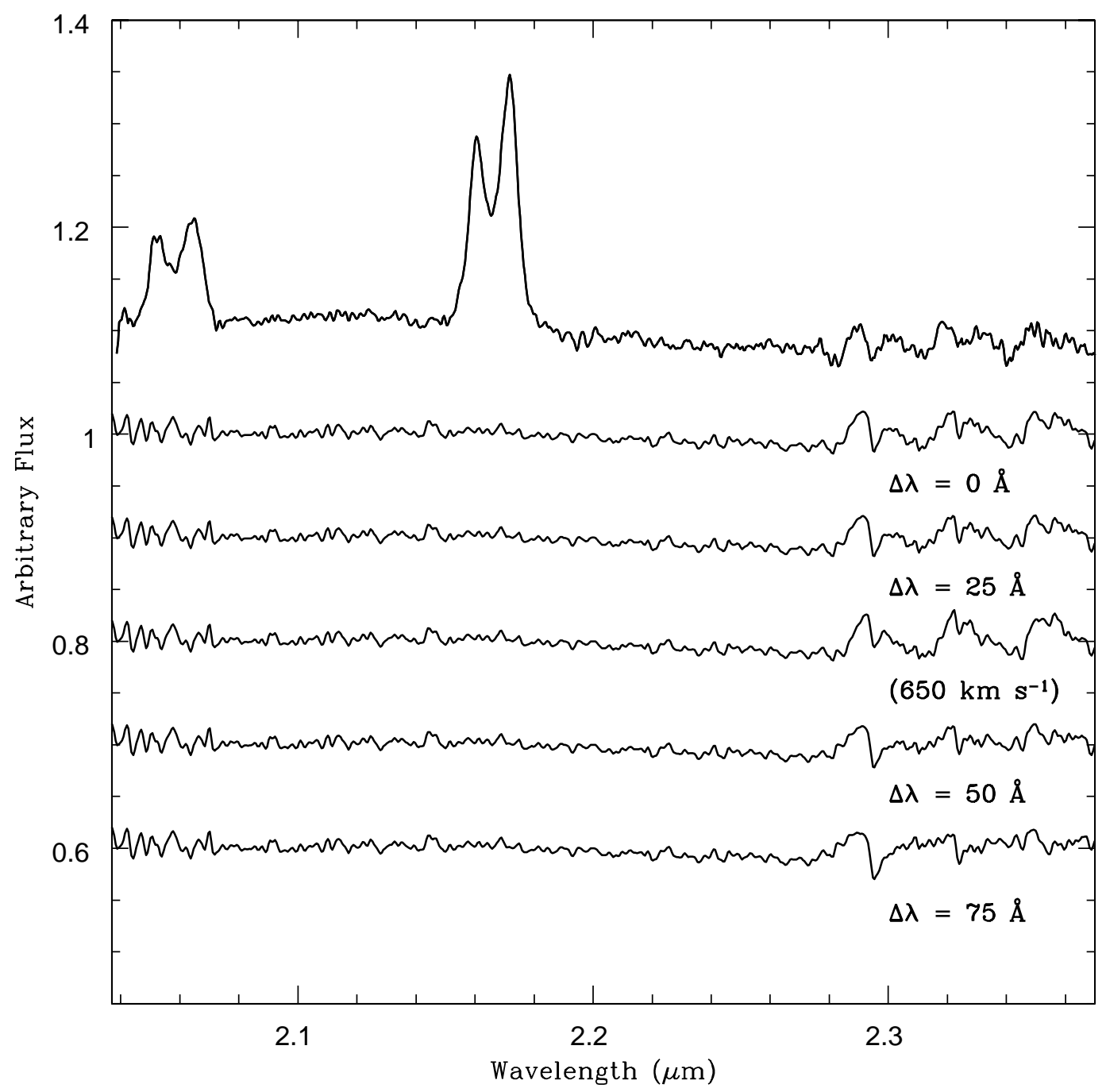

Fig. 6. - Models where we have added a CO emission line spectrum to the L2 dwarf data. The first model spectrum plotted below that of WZ Sge is a single CO emission line spectrum broadened by a Gaussian with a velocity of $1300 \mathrm{~km} \mathrm{~s}^{-1}$ ( " $\Delta \lambda=0 \AA$ ") added to the L2 dwarf spectrum. Clearly, this spectrum closely matches the observations and suggests a normal CO feature in the donor star of WZ Sge. Below that spectrum is plotted the addition of two CO emission line spectra that have had their peaks shifted by $\pm 25 \AA$, and then added to the spectrum of Kelu 1 . At the $1300 \mathrm{~km}$ $\mathrm{s}^{-1}$ velocity broadening used here, there is very little difference between this model, and the single $\mathrm{CO}$ emission line model. If we cut the velocity broadening in half $\left(650 \mathrm{~km} \mathrm{~s}^{-1}\right)$, but keep the \pm 25 $\AA$ separation between the two CO spectra, we get a model that does a much poorer job at matching the observations. Models with larger separations of the CO emission peaks cannot reproduce the observations. 


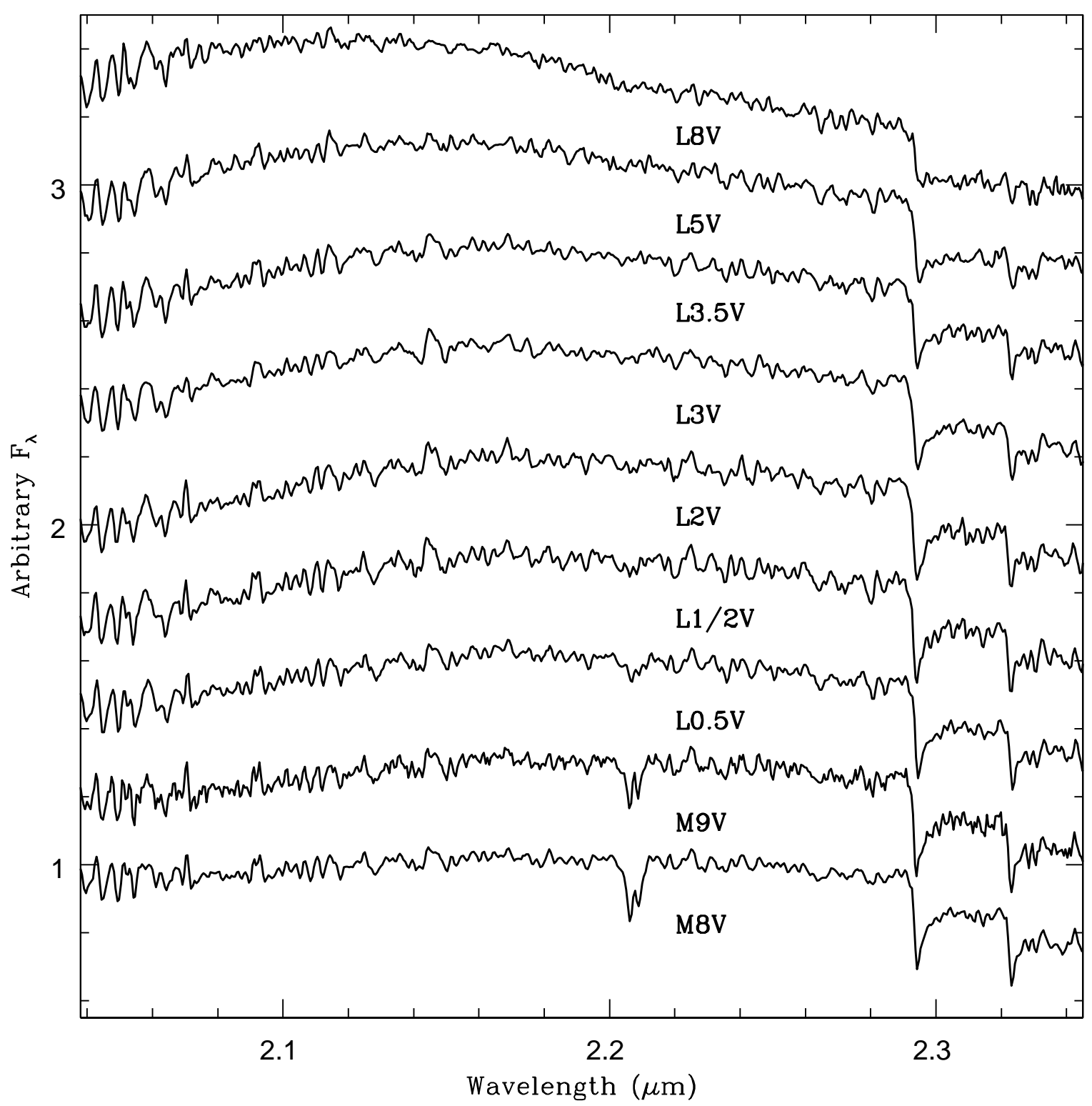

Fig. 7.- A $K$-band spectral sequence of objects near, and below, the stellar/sub-stellar boundary (data obtained from the IRTF Spectral Library). For spectral types earlier than L2, the Na I doublet at $2.2 \mu \mathrm{m}$ is apparent. For the latest $\mathrm{L}$ dwarfs, the ${ }^{12} \mathrm{CO}_{(3,1)}$ feature (at $2.322 \mu \mathrm{m}$ ) becomes very weak. 